REVISTA ECONOMÍA

Vol. 70, N. ${ }^{\circ}$ II2 (noviembre 20I8), I2I-I36

\title{
LOS NÚMEROS TAMBIÉN MIENTEN: SUBEMPLEO Y ESTADÍSTICA LABORAL EN EL ECUADOR
}

\author{
DIEGO CARRIÓN SÁNCHEZ \\ Universidad Central del Ecuador \\ Recepción de manuscrito: 17 de septiembre de 2018 \\ Aceptación versión final: 26 de octubre de 2018
}

\begin{abstract}
RESUMEN En 2014, el Gobierno de turno realizó a través del Instituto Ecuatoriano de Estadística y Censos (INEC) un cambio de metodología en el cálculo de los indicadores de condición laboral en Ecuador, con el fin, se dijo, de seguir directivas provenientes de la Organización Internacional del Trabajo (огт). Sin embargo, una revisión exhaustiva de los cambios operados y la reconstrucción técnico-conceptual de la categoría subempleo, parecen mostrar que otros intereses impulsaron dichos cambios. Una de las consecuencias más importantes del cambio metodológico es que pone en cuestión la hipótesis del subempleo estructural como principal característica del mercado laboral ecuatoriano.
\end{abstract}

PALABRAS ClAVE Subempleo, subempleo estructural, empleo adecuado, ingreso, jornada laboral, deseo y disponibilidad de trabajar más horas.

ABSTRACT In 2014 the Government in turn carried out through Ecuadorian Institute of Statistics and Census (INEC, by its initials in Spanish) a change of methodology in the calculation of the employment status condition in Ecuador, with the aim it was said, of following directives from the International Labor Organization (ILO). However, a thorough review of the changes made and the conceptual technical reconstruction of the underemployment category seem to show that other interests prompted these changes. One of the most important consequences of the methodological change is that it calls into question the hypothesis of the structural underemployment as the main characteristic of the Ecuadorian labor market.

KEYWORDS Underemployment, estructural underemployment, adequate employment, salary, workday.

JEL CODES J81, J82, J88, J30, J21, J22, J23, O17.

\section{INTRODUCCIÓN}

El presente artículo tiene como objetivo aportar a la crítica y construcción de la estadística laboral en el Ecuador. Se propone que dichas mediciones no pueden apuntar solo al cálculo empresarial en el mercado de trabajo, sino que deben ser útiles para entender la situación social de los y las trabajadoras. El análisis se concentra en la categoría subempleo e intenta 
visibilizar las consecuencias del cambio metodológico operado en la estadística del trabajo en 2014 por el INEC.

En la primera sección se discuten los sentidos del concepto subempleo y su vínculo con el modelo de desarrollo ecuatoriano. En la segunda se lo ubica en el contexto de las normas internacionales de la estadística laboral. En la tercera parte se explica el diseño previo al cambio y en la cuarta se analiza críticamente el cambio metodológico operado por el gobierno de Rafael Correa. En la quinta sección se hace una propuesta alternativa provisional para la medición del subempleo en Ecuador y en la sexta se muestran los resultados de su aplicación.

\section{LA CATEGORÍA SUBEMPLEO}

Tanto por su definición técnico conceptual, como por su uso social, la categoría subempleo en el Ecuador tiene al menos dos acepciones. Por un lado, alude al grado de ocupación de la fuerza de trabajo por parte del aparato productivo privado y público; en el sentido de relacionar la demanda de trabajo de los empresarios con la oferta. Por otro lado, subempleo es una medida de la calidad del empleo, es decir, de las condiciones laborales: ingreso, estabilidad, grado de precariedad. El término ha sido usado para dar cuenta del papel del empresariado y en menor medida del Estado, en la generación de empleo y en la calidad del mismo.

En este sentido, ha sido útil para sostener la hipótesis que el desempleo no es el principal problema de las economías dependientes como la ecuatoriana, pues las personas que no encuentran trabajo se inventan alternativas económicas que les permiten sobrevivir - comercio informal, artesanía, prestación precaria de servicios como vigilancia o limpieza de autos, preparación de alimentos, etc.- (Callinicos, 2011). El principal problema en materia laboral de estas economías es el subempleo estructural (Larrea, 2016), es decir, el hecho que más de la mitad de la población no encuentre un trabajo que le garantice los derechos mínimos de un empleo decente y tenga que laborar de manera precaria.

Este tipo de subempleo es estructural porque no depende de la voluntad o el mérito de las personas, sino de la imposibilidad de tener acceso a los medios de producción debido a la enorme concentración privada de los mismos. En Ecuador, el 60,7\% de la tierra disponible es propiedad del 6\% de las Unidades Productivas Agrícolas (UPAS) más ricas, mientras el 75,5\% de las UPAS más empobrecidas posee solo el 11,8\% de la tierra disponible (Carrión, 2012). La concentración de capital podría llegar a $0,96^{1}$ en la escala de Gini (Larrea y Green, 2015). Y la concentración de mercados en sentido monopólico u oligopólico es muy alta (Superintendencia de Control de Mercados, 2013).

Existen restricciones estructurales para realizar nuevas inversiones debido a que el capital está concentrado: el trabajador desocupado o subempleado no dispone de capital y las líneas de crédito resultan inalcanzables o impagables. La concentración de mercados no solo impide un giro de negocio más lucrativo, sino que imponen barreras de inversión más altas, es decir, que para ingresar a la competencia con relativas posibilidades de éxito se debe disponer de capitales cada vez mayores. ${ }^{2}$

Esta realidad es propia de economías primario exportadoras donde los sectores núcleo de la acumulación capitalista emplea a un número reducido de trabajadores, - en el Ecuador, el 
petróleo emplea solo al 0,5\% de la población económicamente activa (PEA) - y se orientan a satisfacer la necesidad externa; economías donde la industria no se encuentra suficientemente desarrollada y la especulación financiera es, a claras luces, más lucrativa (Carrión y Sánchez, 2014); mientras, gran parte de la población se queda fuera de los núcleos de acumulación y fuera de los círculos de la propiedad privada de los medios de producción.

El concepto subempleo, categoría de la variable condición de actividad construida en la estadística laboral del INEC, hacía visible esa realidad. Sin embargo, el gobierno de Rafael Correa decidió en 2014 hacer su segundo ${ }^{3}$ cambio metodológico a la Encuesta Nacional de Empleo Desempleo y Subempleo Urbana y Rural (ENEMDUR), que transformó dicha categoría quitándole el poder semántico que hasta entonces tenía. Como justificación se dijo que el cambio respondía a recomendaciones realizadas por la oIT en la decimonovena Conferencia Internacional de Estadísticas del Trabajo (CIET 19), realizada en 2013 (Castillo, 2015). ¿Fue este cambio metodológico una operación técnica ajustada a las recomendaciones y objetivos de la OIT, o fue una operación política?

\section{LAS ORIENTACIONES DE LA OIT SOBRE SUBEMPLEO}

La decimosexta Conferencia Internacional de Estadísticas del Trabajo (CIET 16), realizada en 1998, era la base para las definiciones técnicas y conceptuales con las que el INEC construía la variable condición laboral que medía el mercado de trabajo en el Ecuador, antes del cambio metodológico del 2014.

La CIET 16 se preocupaba explícitamente por visibilizar a trabajadores que en los países periféricos no podían ser clasificados como empleados plenos o como desocupados, pues, sin tener un empleo adecuado, buscaban la manera de sobrevivir inventando actividades económicas o aceptando trabajos parciales y precarios que los sacaran de la desocupación (Laurie, 1998). Por ello, la OIT, a través de la resolución de la CIET 16, recomendaba a todos los países la presentación de cifras sobre subempleo por insuficiencia de horas de trabajo, pero ampliando las mediciones para incluir situaciones de empleo inadecuado. Es decir, que se consideraba necesario no medir únicamente la utilización de la fuerza del trabajo en el mercado - la relación oferta demanda de trabajo-, sino también la calidad del trabajo a través del llamado «empleo inadecuado» (16. ${ }^{\mathrm{a}}$ CIET, 1998).

Si bien la oIT recomendaba medir la relación oferta-demanda de trabajo y el empleo inadecuado de manera separada, Ecuador incluye dentro de la propia definición de subempleo indicadores de mercado e indicadores de calidad construyendo un concepto de doble acepción. El subempleo estará definido por tres variables, dos vinculadas a la medición del grado de utilización de la fuerza de trabajo en el mercado: horas trabajadas y deseo y disponibilidad de trabajar más horas; y una variable que funciona como proxi a la calidad del trabajo que es el ingreso. Esta definición permitía interpretar hasta el 2014 el porcentaje de subempleados, como el conjunto de trabajadores que el aparato productivo no lograba emplear de manera suficiente y adecuada.

Un cambio de dirección empieza a gestarse con la CIET 19. La resolución de esta conferencia, más allá de la retórica que es común en este tipo de documentos, apunta a adecuar la estadística laboral internacional para ser funcional a la construcción del Sistema de Cuentas 
Nacionales del 2008 (SCN 2008) auspiciado por el Fondo Monetario Internacional (FMI), el Banco Mundial (BM), las Organización de las Naciones Unidas (ONU), entre otras organizaciones internacionales. Esto implica limpiar de cuestiones valorativas o cualitativas al concepto de subempleo, para convertirlo enteramente en un indicador del mercado de trabajo que mida la incompatibilidad entre oferta y demanda laborales.

Dos son los énfasis técnicos para la construcción de la estadística del trabajo recomendada a todos los países: i) eliminar toda connotación cualitativa de la subutilización del trabajo y ii) cuantificar otras formas de trabajo necesarias para la construcción del sCN como el trabajo de autoconsumo, trabajo en formación no remunerado o el trabajo voluntario.

El InEC cambia la metodología siguiendo supuestamente estos lineamientos internacionales, pero en realidad no cumple con ninguno de los dos objetivos planteados por la CIET 19: i) el Ingreso sigue siendo una variable para definir si una persona es o no subempleada, manteniendo el carácter cualitativo de la medición; y ii) no se las reagrupa en las categorías definidas por la CIET 19, sino que se crean nuevas categorías, que como veremos, responden a otros intereses, propios del gobierno y no a los objetivos técnicos de la oIT.

En relación a la medición de otras formas de trabajo, el INEc hasta la fecha, no contabiliza ni difunde información sobre ellas. ¿Cuáles fueron, entonces, los cambios metodológicos realizados y cuáles sus objetivos?

\section{EL DISEÑO PREVIO A 2014}

Antes de abordar los cambios se hará un breve repaso sobre el diseño metodológico de la categoría subempleo en la ENEMDUR antes del 2014. Cabe aclarar que este diseño previo incluía ya importantes distorsiones a la medición del subempleo que se conservarán y sumarán a las distorsiones del cambio metodológico de 2014.

Los siguientes criterios para definir si un trabajador o trabajadora es subempleado no han sido cambiados y se mantienen vigentes a la fecha: i) si su salario está por debajo del umbral de salario adecuado fijado por cada país — en Ecuador se considera salario adecuado al Salario Básico Unificado (sBU) -; ii) si las horas trabajadas efectivamente en el período de referencia de la encuesta ${ }^{4}$ es menor a la jornada laboral legal de 8 horas; y iii) si está dispuesto y disponible a trabajar más horas de las que trabajó en el periodo de referencia.

Según el diseño de la variable condición de actividad para 2007, vigente hasta 2014, existían los tipos de subempleo según se combinen los tres criterios anteriores. Sobre el número de subempleados calculados en base de esta metodología, incidirá también la edad mínima a partir de la cual se considera a alguien trabajador. Entre 2007 y 2014 - y antes de dicho período-, la edad mínima para ser contabilizado era 10 años, lo que permitía incluir el trabajo infantil dentro de la subocupación.

\section{EL CAMBIO METODOLÓGICO DE 2014 Y SUS CONSECUENCIAS}

El análisis del cambio metodológico se dividirá en dos secciones. La primera aborda el tratamiento que el gobierno de Rafael Correa dio a los criterios para la definición del subempleo: 
Tabla 1: Ingreso mensual

\begin{tabular}{lc}
\hline COMPONENTES DEL INGRESO DEL HOGAR & USD \\
\hline SBU & 375 \\
\hline+ Pago mensual por los décimos & 62,5 \\
\hline = Nombre de primer perceptor & 437,5 \\
\hline Salario de segundo perceptor (60\% del SBU) & 262,5 \\
\hline Ingreso de hogar con 1,6 perceptores & 700 \\
\hline
\end{tabular}

Elaboración: autor.

ingreso, jornada, deseo y disponibilidad, con lo que consigue sobreestimar el empleo adecuado. La segunda sección tratará sobre el reagrupamiento de trabajadores subempleados bajo nuevas categorías, cuyo objetivo es subestimar el subempleo.

CRITERIOS PARA DEFINIR EL SUBEMPLEO

El primer cambio operado fue el cambio de la edad mínima en la estadística laboral que afecta a la magnitud del subempleo. A partir de 2014, los cálculos presentados al público se hacen considerando la población de 15 años o más, cuando antes se consideraba la población de 10 años y más. De esa forma se deja fuera de la estadística de subempleo a buena parte de los trabajadores infantiles que laboran mayormente en condiciones precarias.

\section{INGRESO}

La OIT libera a los países para fijar el límite de lo que se considera salario adecuado según sus condiciones nacionales (16. $\left.{ }^{\mathrm{a}} \mathrm{CIET}, 1998\right)$. Ecuador define como límite el salario básico unificado o salario mínimo, que es un umbral muy bajo y distorsiona la estadística laboral, pues sobreestima el trabajo adecuado. Muchos trabajadores que no logran satisfacer sus necesidades básicas con su salario, aparecen como empleados plenos, con salario adecuado.

Rafael Correa no estableció este criterio, pues se venía usando de antes. Sin embargo, su equipo aportó una justificación novedosa a su uso, argumentando que los hogares tipo en Ecuador tienen 1,6 perceptores y que su ingreso sería suficiente para cubrir la canasta básica familiar (СвF) que asciende a USD 705,4.5 Para ello, deberían cumplirse las siguientes condiciones: i) el primer perceptor recibe el salario mínimo (USD 375) y sus patrones le pagan el décimo tercero y el décimo cuarto sueldo; ii) el primer perceptor mensualiza el pago de sus décimos recibiendo en total la suma mensual de USD 437,5 (salario básico + décimos mensualizados); y iii) el segundo perceptor recibe un mínimo de USD 262,5 equivalente al 60\% de un salario básico.

De esta manera, suman USD 700 de ingreso mensual (ver Tabla 1). Sin embargo, según la ENEMDUR, en Ecuador $61 \%$ de los hombres no logra completar un ingreso equivalente al de primer perceptor y al menos $46 \%$ de las mujeres no alcanza un sueldo de segundo perceptor. Además, 51\% de los hombres no recibe decimotercero ni decimocuarto sueldo, por lo que tampoco podrían mensualizarlos para alcanzar los ingresos necesarios para cubrir la CBF. 
Los gastos de educación $\left(14 .^{\circ}\right)$ y fin de año $\left(13 .^{\circ}\right)$, son rubros que no consienten ser prorrateados mes a mes, pues tienen destino previo y no trasladable. Es decir, el sueldo básico en el Ecuador alcanzaría para cubrir la canasta básica, según el Gobierno de R. Correa, pero sin cuadernos, ni lápices, ni regalos de Navidad para los hijos de los trabajadores. La definición de perceptores que utiliza el INEC es defectuosa: define como perceptor a toda persona que reciben un ingreso de cualquier fuente y origen (INEC, n. d.), por lo que no garantiza ni periodicidad de la percepción, ni que la cantidad recibida equivalga al 60\% de un salario básico. Por otro lado, este supuesto naturaliza el hecho que la mujer tenga un salario menor al hombre y considera normal que la doble jornada laboral y el trabajo reproductivo no remunerado corresponda a la mujer.

Lo dicho permite considerar de manera seria la hipótesis que más de la mitad de los hogares ecuatorianos no pueden pagar la CBF mes a mes; que el supuesto de 1,6 perceptores no es adecuado para establecer un límite normativo del salario y, por tanto, no se puede confiar que el salario básico unificado sea un buen indicador para evaluar el empleo adecuado, pues, resulta un umbral muy bajo que no refleja la realidad de la mayoría de los hogares ecuatorianos. Aquí se registra la primera sobreestimación del pleno empleo o trabajo adecuado, puesto que se contabiliza personas que ganando el salario mínimo no logran satisfacer sus necesidades ni las de su familia.

\section{JORNADA LABORAL}

El INEC considera como jornada adecuada aquella que es igual o mayor a ocho horas diarias, por tanto, tendrían una jornada inadecuada solo los trabajadores con jornadas menores a la legal. De esta forma se sobreestima por segunda vez el empleo adecuado, pues se consideran trabajadores plenos a personas que son sobreexplotadas al tener jornadas más largas que la legal en combinación con salarios que no cubren la reproducción de sus necesidades básicas (Marini, 2008).

\section{DESEO Y DISPONIBILIDAD}

El deseo es un indicador subjetivo que indica la voluntad personal de trabajar más horas; se entiende que dicho deseo está relacionado con el fin principal de obtener mayores ingresos para satisfacer necesidades. La disponibilidad es un indicador objetivo que muestra cuándo una persona, teniendo el deseo de trabajar más horas para obtener mayores ingresos, no puede hacerlo por distintas razones que escapan a su voluntad: no encuentra otro trabajo, tiene ocupaciones de cuidado o reproducción en el hogar, tiene mucho cansancio por la demanda de energía de su trabajo principal, entre otros.

El INEC utiliza los criterios de deseo y disponibilidad como si fueran uno solo referido a la voluntad o deseo, es decir, si una persona desea trabajar más horas, pero no está disponible, aparece en la estadística como si no deseara trabajar más. Lo hace por dos motivos. En primer lugar, porque las preguntas del cuestionario de la encuesta no proporcionan la información suficiente para un tratamiento adecuado del criterio disponibilidad; $y$, en segundo lugar, porque en el afán de sobreestimar el pleno empleo, es más conveniente priorizar el criterio deseo como indicador de la voluntad de los trabajadores. Así, toda falla o limitación del 
Figura 01. Impacto de la reforma metodológica sobre la categoría subempleo

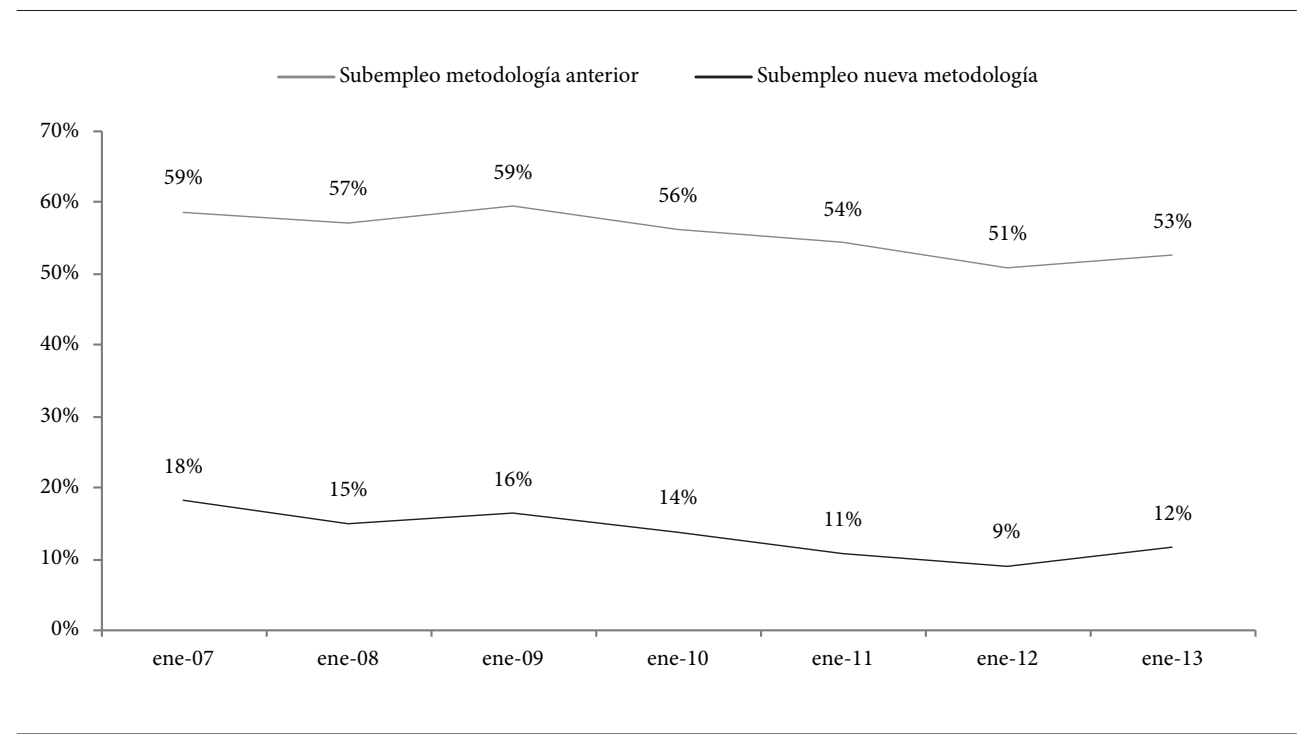

Fuente: (Castillo, 2015). Elaboración: propia.

aparato productivo - empresarios + Estado- quedan ocultos tras la supuesta falta de voluntad de los trabajadores para trabajar más. Por ejemplo, una mujer que debido al trabajo de cuidado de sus hijos y/o personas mayores, desea, pero no puede por sus ocupaciones, trabajar más horas; o un trabajador al que se le exige mayor intensidad de trabajo dentro de la misma jornada de ocho horas y estará demasiado cansado para poder trabajar más. Ambos casos ya no serán registrados como subempleados.

Se incluye como personas que no desean trabajar más, a todas las que no puedan hacerlo por razones estructurales. No se considera la posibilidad de desear trabajar más horas, pero no estar disponible por condiciones fuera de la voluntad.

Pensar que una persona que no tiene un ingreso suficiente para sostenerse a sí misma y su familia, no está dispuesto a trabajar por pura voluntad es tener como supuesto de partida la idea que las personas son resignadas u ociosas «por naturaleza», siendo la laboriosidad y el trabajo una virtud extraña o artificial que debe propiciarse. Este ha sido un argumento recurrente del liberalismo económico para justificar la desigualdad sugiriendo que los ricos son ricos porque trabajan más y los pobres son pobres por ser ociosos. Lo que es claramente falso, pues la desigualdad es resultado de la mala distribución de la riqueza, el ingreso y los medios de producción (Eagleton, 2010; Bauman, 2014; Dubet, 2016).

Respaldando este error, el Gobierno de R. Correa decide crear una nueva categoría: «Otro empleo no adecuado», que incluye a estas personas que tienen salarios menores al básico, pero asoman como no dispuestas a trabajar más horas - aunque esto no responda a su deseo, sino a las fallas estructurales que no les permiten estar disponibles-. Estas personas dejarán de ser cuantificadas como subempleadas porque formarán parte de esta nueva categoría. 
REAGRUPAMIENTO DE TRABAJADORES EN NUEVAS CATEGORÍAS

Los trabajadores que antes se clasificaban en dos grupos, subempleo visible o por tiempo y otras formas de subempleo, ahora se descomponen en cuatro grupos: subempleo por tiempo, subempleo por ingresos, otra forma de subempleo y empleo no remunerado. El subempleo estará compuesto por subempleo por tiempo y por ingreso, mientras las otras dos categorías ya no serán contabilizadas como subempleo.

SUBEMPLEO

La única directriz de la CIET 19 que el Gobierno de R. Correa aplica es la que define al subempleo en función del deseo y disponibilidad de trabajar más horas exclusivamente. Se lo hace a pesar de los errores en esta medición que se explicaron en la sección anterior. Se considera subempleadas a las personas que con ingreso adecuado o inadecuado, con jornada adecuada o inadecuada, desean y están disponibles a trabajar más horas. El subempleo se dividirá en: i) subempleo por tiempo, y ii) subempleo por ingreso.

En este punto, el Gobierno decide subestimar el subempleo por ingreso que es el componente más grande del subempleo en el país. Se subestima cuando se decide sumar a aquellas personas que tienen ingreso inadecuado y jornada inadecuada, las dos condiciones juntas, a subempleo por tiempo y no a subempleo por ingresos. El impacto es fuerte y se analiza más adelante.

\section{OTRO EMPLEO NO ADECUADO}

Se crea la categoría otro empleo no adecuado, la cual en la resolución de la CIET 19 no aparece mencionada. Según el Gobierno de R. Correa esta categoría estaría compuesta por los siguientes subgrupos: i) personas con ingreso no adecuado, con jornada adecuada, que no tienen deseo ni disponibilidad de trabajar más horas; y ii) personas con ingreso no adecuado, con jornada no adecuada, que no tienen deseo ni disponibilidad de trabajar más horas.

Una primera observación importante es que la creación de la categoría otro empleo no adecuado, también contribuye a subestimar el subempleo por ingresos, pues los dos grupos que la componen tienen ingresos menores al sBU y, sin embargo, no se suman en dicha categoría.

En segundo lugar, hay que decir que la única función de esta nueva categoría es disminuir el peso que el subempleo representa en el mercado laboral ecuatoriano, pues incluye a personas que supuestamente no tienen deseo ni disponibilidad de trabajar más horas; pero oculta que en ese grupo contabiliza a personas que desean trabajar aunque no tienen disponibilidad para hacerlo - como una mujer dedicada al cuidado o un obrero agotado que no pueden trabajar más horas-. Quien desea trabajar más, por necesidad, es sin duda un subempleado, aunque no esté disponible debido a razones que escapan a su voluntad.

\section{EMPLEO NO REMUNERADO}

Personas que no reciben ningún tipo de remuneración por su trabajo, con o sin jornada adecuada. Para su medición, resulta irrelevante la pregunta sobre deseo y disponibilidad. No se refiere al trabajo reproductivo no remunerado de las mujeres que se ocupan de su hogar, 
Figura 02. Subempleo y empleo adecuado (porcentaje PEA) (junio 2017). Escenarios comparativos.

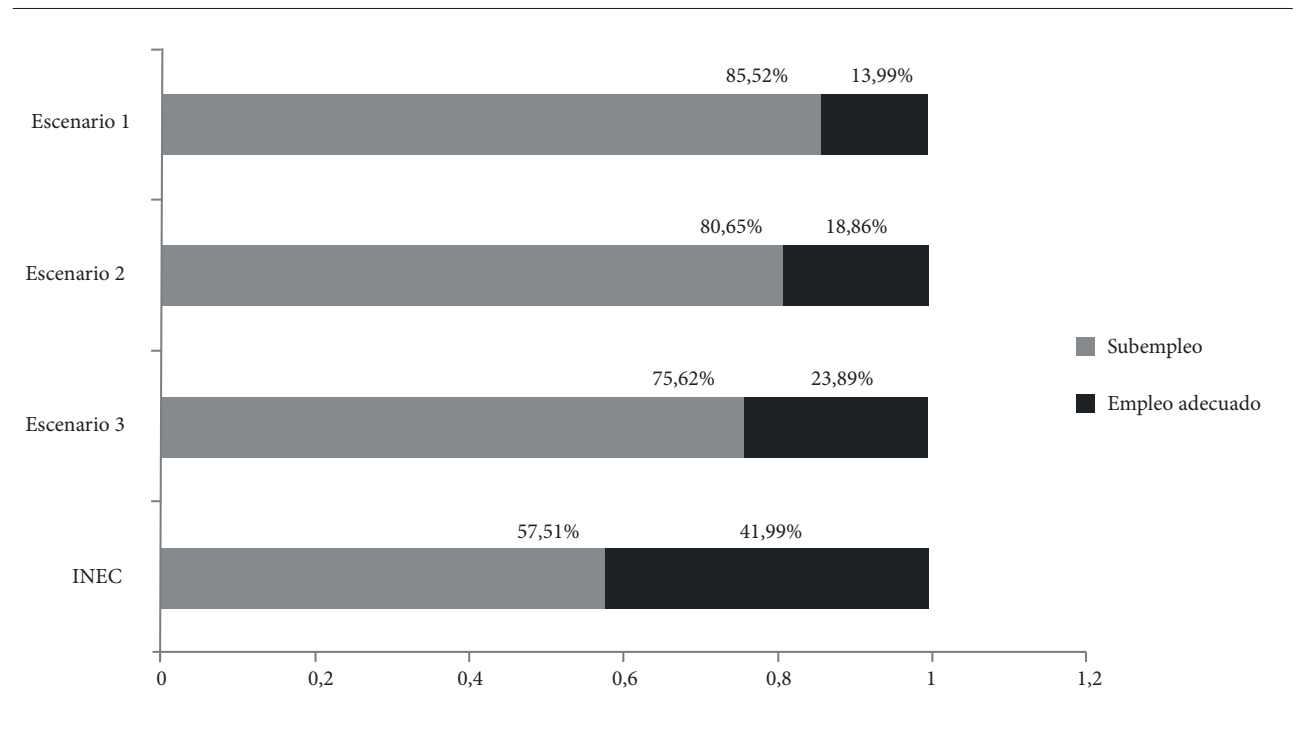

Fuente: INEC-ENEMDU (junio 2017). Elaboración: propia.

pues éste es registrado torpemente por el INEC dentro de la población económicamente inactiva (PEI). Antes, el trabajo no remunerado se sumaba en bloque a la masa de subempleados, ahora se presenta como una categoría que se refiere a tres tipos de trabajadores: i) trabajadores del hogar no remunerados: hijas, abuelas, otros familiares que trabajan en el hogar que no se identifican como «amas de casa»; ii) trabajadores no remunerados en otro hogar: personas que ayudan en un hogar diferente al suyo sin remuneración; y iii) ayudantes no remunerados de asalariados y jornaleros: normalmente vinculados a tareas agrícolas y unidos al asalariado o jornalero por lazos familiares.

Todos los cambios mencionados no se discutieron ni analizaron públicamente; tampoco se informó debidamente a la población (Villacís, 2016). Si en un primer momento la información se presentaba agregada en la categoría empleo inadecuado que sumaba subempleo, otro empleo no adecuado y trabajo no remunerado a partir de marzo de 2016, se deja de utilizar la categoría agregada y se presenta los datos individualmente (Villacís, 2016), lo que demuestra la intensión de confundir a la población y beneficiarse de la difusión de un dato sobre subempleo a todas luces falso, que daría a entender que la hipótesis del subempleo estructural en Ecuador estaría superada (ver Figura 1).

Se presentó en promedio una reducción del subempleo del $42 \%$ operada únicamente en el papel con importantes efectos políticos: se minimizó un indicador que subraya la responsabilidad del empresariado y el Estado en un mercado de trabajo deprimido y de mala calidad.

\section{PROPUESTA ALTERNATIVA PROVISIONAL}

En este apartado se realiza una propuesta metodológica provisional para el cálculo de la categoría subempleo que busca respetar el doble uso semántico de la categoría, tal como ha sido 
tradicional en el país: como medida del mercado de trabajo y como índice de calidad del empleo. Se dice que es provisional porque aún queda mucho que mejorar en la medición de la calidad y nivel de vida de los trabajadores, tanto en la recolección de datos como en el análisis. Por tanto es perfectible y pretende únicamente plantear un reto para la profundización y mejoramiento de las estadísticas laboral. No obstante, se confía que sus resultados son una buena aproximación a la realidad del mercado laboral en el Ecuador.

La explicación que sigue alude únicamente a las cuestiones conceptuales de la metodología de cálculo. Pero debe saberse que detrás de cada concepto hay un diseño técnico de sintaxis o conjuntos de expresiones lógicas que, aplicadas a la base de datos de la ENEMDUR en el programa estadístico sPss, agrupa y cuantifica cada categoría presentada. Se procede de esta manera para facilitar la lectura y el análisis de los cambios realizados, mejorando así la interpretación de los datos que se presentan en la próxima sección. Se presentarán primero los cambios propuestos para los criterios de medición de subempleo y luego la organización de las categorías.

\section{CRITERIOS PARA DEFINIR EL SUBEMPLEO}

\section{INGRESO}

Considerando que el umbral adecuado de ingreso elegido es muy bajo como para dar cuenta de la calidad del empleo en el Ecuador, se propone calcular el subempleo y sus categorías en tres escenarios que consideran diferentes niveles de ingreso con la finalidad de compararlos con los resultados presentados por el INEC. Escenario 1: se considera como salario adecuado al valor de la canasta básica familiar (USD 706,40); escenario 2: se calcula con un salario adecuado equivalente a USD 600,00, que corresponde al ingreso que debería tener el primer perceptor

más el o,6 del ingreso que debería tener el segundo perceptor antes de los sobresueldos; y escenario 3: se considera como salario adecuado el valor de la canasta familiar vital (USD 507,47). ${ }^{6}$

\section{JORNADA LABORAL}

Se propone incluir como trabajadores con jornada inadecuada a aquellos que trabajan más de ocho horas debido a exceso de trabajo o clientes, o porque consideran que es necesario trabajar más de las 40 horas para cubrir sus necesidades. Se excluyen aquellos que, trabajando más de las 40 horas, cobraron horas extras.

\section{DESEO Y DISPONIBILIDAD}

Solo si una persona tiene deseo y disponibilidad de trabajar, el INEC la clasifica como subempleada. Si tiene deseo, pero no disponibilidad, la clasifica como otro empleo no adecuado, subestimando de esa forma el subempleo. Por ello se propone agregar a la categoría subempleo personas que, teniendo el deseo de trabajar más, no están disponibles por causas estructurales. Se logra, así, que en el cálculo de los indicadores de empleo adecuado y subempleo la voluntad no tenga un peso exagerado; se pone el peso adecuado sobre las condiciones materiales de expropiación y desamparo que impide a la gente trabajar más y se reconoce el trabajo 
Figura 03. Composición del empleo como porcentaje de la PEA (junio 2017).

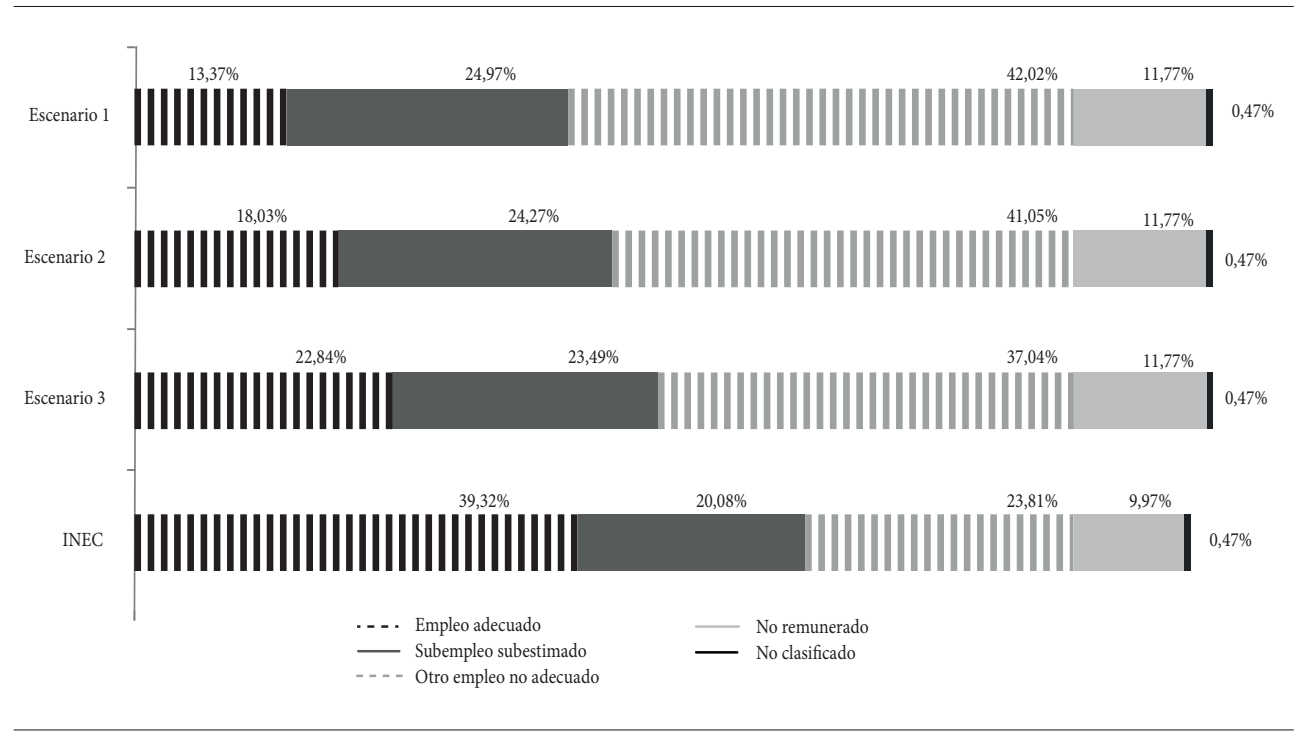

Fuente: INEC-ENEMDU (junio 2017). Elaboración: propia.

de reproducción y cuidado. Para lograrlo se hace necesario reagrupar a los trabajadores en las categorías del subempleo. Tarea que se realiza en la siguiente sección.

\section{REAGRUPAMIENTO DE TRABAJADORES EN CATEGORÍAS ALTERNATIVAS}

El reagrupamiento propuesto bebe de dos fuentes para conformar las nuevas categorías relacionadas al subempleo. La primera fuente son los trabajadores que en la metodología de 2014 pertenecen a las categorías subempleo, otro empleo no adecuado y empleo no remunerado. La segunda fuente, de donde proviene un porcentaje importante de trabajadores reclasificados aquí como subempleados, es la categoría empleo adecuado.

Desde la categoría empleo adecuado se traslada a subempleo los siguientes grupos de trabajadores: i) trabajadores con jornadas superiores a la legal sin pago de horas extras, se traslada a subempleo por tiempo; ii) trabajadores con ingreso no adecuado, según los tres escenarios propuestos de umbral de ingreso, se trasladan a subempleo por ingreso; y iii) trabajadores que desean trabajar más horas, pero no están disponibles por razones estructurales, se trasladan a otro empleo no pleno. Para los fines pertinentes subempleo estará compuesto como se describe en las siguientes fórmulas:

$$
\begin{aligned}
& \text { SUBEMPLEO = subempleo por horas + }(\text { subempleo por ingreso + otro empleo no pleno })+\text { em- } \\
& \text { pleo no remunerado. } \\
& \text { SUBEMPLEO }=\text { subempleo por horas }+ \text { subempleo por ingreso + empleo no remunerado. } \\
& \text { SUBEMPLEO }=\text { subempleo por horas }+ \text { subempleo por ingreso. }
\end{aligned}
$$

El subempleo por ingreso debe entenderse como la suma del subempleo por ingreso y otro empleo no pleno, debido a que la diferencia entre las dos categorías es solo la disponibilidad o no 
a trabajar más horas; mientras la similitud entre ambas es que las personas agrupadas tienen deficiencia de ingresos. El INEC ha procurado ocultar el subempleo por ingreso. Existen trabajadores que tienen solo insuficiencia por ingreso, otros que tienen solo insuficiencia por horas, pero hay trabajadores que tienen insuficiencia de ingresos y horas. Se enfrenta la decisión de ubicar este tercer grupo en una de las dos categorías disponibles: subempleo por ingreso o por horas. El INEC opta por ubicar a las personas con «insuficiencia de horas e ingreso» en la categoría subempleo por horas. Considerando que estos trabajadores con doble insuficiencia representan el grueso de los subempleados por ingreso, dicha decisión implica ocultar la realidad.

Por esa razón, se propone corregir ese desacierto y se suma a las personas con insuficiencia de horas e ingresos en la categoría subempleo por ingresos. También se propone sumar a la categoría subempleo a los trabajadores no remunerados, debido a que al no recibir ningún tipo de pago tienen insuficiencia de ingreso e, independientemente de la suficiencia o no de la jornada laboral o la disponibilidad de trabajar más horas, resultan una de las categoría más precarizada y explotada en el mercado.

\section{LA SITUACIÓN DEL SUBEMPLEO EN EL ECUADOR}

Según los cálculos del INEC, solo existiría un 20\% de subempleados en el Ecuador. Este dato está claramente subestimado. Si sumamos los grupos humanos que tradicionalmente componían esta variable, previo al cambio metodológico del año 2014, más la categoría inventada por el gobierno de Rafael Correa - otro empleo no adecuado- encontraremos que, con los mismos cálculos y supuestos asumidos por el INEC, el subempleo en Ecuador ascendería al menos al 55\% de la PEA. Pero como se mostró en secciones anteriores incluso este último dato está subestimado debido a los criterios utilizados, principalmente el bajo umbral de ingreso considerado «adecuado».

Para dimensionar el peso del subempleo en el Ecuador se propone observar los resultados de la propuesta metodológica alternativa que se exponen a continuación. La siguiente figura considera los escenarios de ingresos propuestos. Lo que varía entre los datos del INEC y los escenarios es resultado de los cambios metodológicos que hemos realizado para corregir el enfoque del InEC (ver Figura 2).

En el escenario 3 - salario equivalente a la canasta familiar vital, USD 507,47-, el subempleo ascendería a $72 \%$ de la PEA. En el escenario 2 - salario equivalente a 1,6 perceptores, USD 600 - el subempleo sería del $77 \%$. Y en el escenario 1 - salario equivalente a la canasta básica familiar, USD 706,4), el subempleo asciende al 82\% de la población económicamente activa. La cifra depende del salario que consideremos como «adecuado». El INEC, como hemos visto, establece un umbral muy bajo - el sBU-, por lo que las cifras de personas consideradas en el subempleo se subestiman dramáticamente.

La amplia diferencia entre los datos del INEC y los datos calculados en nuestros escenarios, se debe principalmente a la inclusión de umbrales de ingreso diferentes. El impacto es tan grande porque aproximadamente un $70 \%$ de los trabajadores tiene ingresos menores a USD 507,47 (escenario 3 ); un $77 \%$ salarios menores a usD 600 (escenario 2 ) y un $82 \%$ salarios menores a USD 706,4 (escenario 3). 
Figura 4. Composición porcentual del subempleo, según INEC y escenarios propuestos (junio 2017)

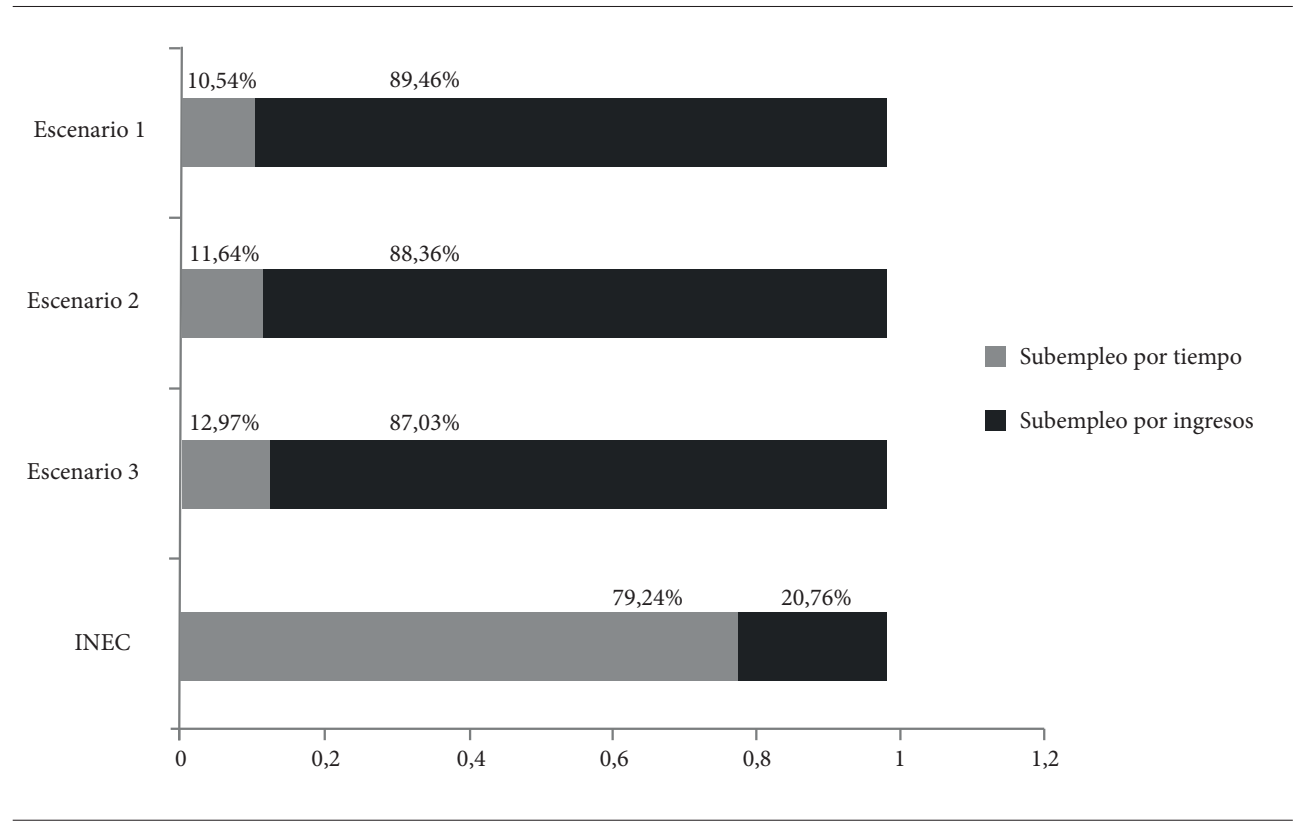

Fuente: INEC-ENEMDU (junio 2017). Elaboración: propia.

Los datos obtenidos sugieren que tomando como fuente al INEC o los escenarios aquí elaborados, más de la mitad de la PEA en Ecuador se encuentra subempleada, lo que confirma la hipótesis de la existencia de subempleo estructural en el país. Esto significa que el aparato productivo, representado principalmente por los empresarios privados, no emplea de manera plena las capacidades de más de la mitad de los trabajadores y, además, que estos tienen condiciones laborales precarias. Según el escenario asumido en cada caso, el nivel de subempleo oscila entre 72 y $82 \%$ de la PEA. El empleo pleno oscilaría apenas entre el 13 y el 23\% de la PEA.

Que un porcentaje tan alto de la población tenga condiciones laborales precarias significa que tiene salarios muy bajos, que trabaja menos o más horas que la jornada legal, que teniendo deseo y/o disponibilidad de trabajar más horas para satisfacer sus necesidades no logra conseguir empleo, o, deseando trabajar más, no puede dedicar más horas al trabajo por condiciones que no dependen de su voluntad. Son subempleados personas que cumple una o varias de estas condiciones (ver Figura 3).

Desde una óptica de calidad, el empleo adecuado se sitúa muy por debajo de lo considerado por el INEC (ver segmento «empleo adecuado» de la Figura 3 ).

Es notable la magnitud del empleo no remunerado (ver segmento «empleo no remunerado»): 11,8\% de la PEA. Principalmente porque éste no refiere al trabajo reproductivo y de cuidado de las mujeres que ha sido «naturalizado» por el INEC al ubicarlo dentro de la población económica inactiva, sino que corresponde a: i) personas que realizan trabajos no remunerados dentro del hogar aunque no sean familiares; ii) hijas/os, tías/os, abuelas/os y otros parientes que trabajan de manera no remunerada en el hogar, pero no se consideran «ama de casa»; 
y iii) personas que ayudan a jornaleros o asalariados sin recibir remuneración. Es decir, un importante porcentaje de trabajadores que realizan trabajos imprescindibles para la reproducción del sistema social, y a pesar de ello, no tienen remuneración por su esfuerzo.

Con la creación de la categoría otro empleo no adecuado, al separar del subempleo a los no remunerados, y con el resto de cambios metodológicos, el INEC continúa la tendencia de vaciar la categoría subempleo para minimizarla. Lo que tiene un efecto en la opinión pública, generando la imagen de una reducción impactante de la cifra. Se lo hace expulsando de la categoría subempleo entre el 48,8\% y el $57 \%$ de los subempleados. Además se busca esconder la magnitud del subempleo por ingreso porque éste indica el grado de explotación del capital sobre el trabajo. Se puede a preciar la composición porcentual del subempleo según el INEC y la composición porcentual de los escenarios propuestos (ver Figura 4). Mientras que para el INEC, el subempleo por ingresos representa solo el $21 \%$ de los subempleados; en los escenarios que corrigen la metodología utilizada, el subempleo por ingresos oscilaría en realidad entre $87 \%$ y $89 \%$ de los subempleados.

\section{CONCLUSIONES}

Los cambios hechos en 2014 por el INEC en la estadística laboral no cumplieron los objetivos de la CIET 19, más bien fueron contrarios a algunas de sus directivas. Al parecer, la intención fue política: subestimar el subempleo y sobreestimar el empleo adecuado para tener cifras positivas que mostrar. Colateralmente se liberó de responsabilidad sobre la escasez de trabajo y su pésima calidad a los empresarios que conforman el aparato productivo privado. Al recomponer la categoría subempleo mediante la metodología alternativa aquí propuesta, se observa que la hipótesis de subempleo estructural en el Ecuador sigue siendo válida.

A pesar de los intentos por ocultar la magnitud del subempleo por ingresos, la aplicación de la metodología alternativa propuesta permite observar que la mayor parte de desempleados son definidos por la insuficiencia de ingresos, lo que habilita pensar que el problema de la apropiación de la riqueza generada por el trabajo por parte de los empresarios - explotación laboral — sigue siendo la causa primordial de la desigualdad social.

Frente a la magnitud del subempleo, es difícil no preguntarse por la eficiencia económica y social de una concentración tan profunda de la riqueza. Dentro del horizonte de posibilidades humanas, ¿es en realidad lo mejor mantener una estructura de propiedad privada que niega el libre vínculo entre trabajo y medios de producción, y lo condiciona a la ambición ilimitada de unos pocos propietarios?

Para finalizar, no está de más decir que no son los números los que mienten, sino aquellas personas que en los distintos grados de la jerarquía estatal y empresarial apoyan la producción de información estadística inocua o apologética. Lejos de una actividad objetiva, la producción estadística está embarrada de subjetividad. En el caso presentado se trata de la subjetividad de quienes detentan el poder y utilizan los números para producir la verdad que conviene a la dominación económica y política. 


\section{NOTAS}

1 Larrea y Green (2015) indican que hay dificultades para acceder a información que permita los cálculos sobre concentración de capital y las fuentes disponibles tienen sus limitaciones; sin embargo, este dato es consistente con varias fuentes.

2 Vale decir que estas limitaciones estructurales son la principal razón para sostener que el voluntarismo propulsor de la «política de los emprendedores» implica una promesa falsa que intenta, a nivel ideológico, culpar a las víctimas de su propia desgracia.

3 El primero fue realizado en 2007.

4 El período de referencia de la ENEMDUR es la semana pasada a la aplicación del formulario de encuesta.

5 Todos los cálculos de esta sección se hacen con datos del INEC a junio de 2017.

6 Datos provenientes del INEC (junio 2017).

\section{REFERENCIAS}

16. ${ }^{a}$ Conferencia Internacional de Estadísticos del Trabajo (CIET). (1998). Resolución concerniente a la medición del subempleo y las situaciones de empleo inadecuado, adoptada en la desimosexta Conferencia Internacional de Estadísticos del Trabajo. Organización Internacional del Trabajo. Recuperado de http://www.ilo.org/global/statistics-and-databases/standards-and-guidelines/resolutions-adoptedby-international-conferences-of-labour-statisticians/wCMs_087489/lang--es/index.htm

Bauman, Z. (2014). ¿La riqueza de unos pocos nos beneficia a todos? Bogotá, Colombia: Editorial Planeta Colombiana s. A.

Callinicos, A. (2011). Contra el posmodernismo. Buenos Aires, Argentina: R y R.

Carrión, D. (2012). La palabra en nuestra orilla. Estructura agraria y modelo de acumulación rural en Ecuador: información para el debate político. Quito, Ecuador: Fundación Rosa Luxemburgo.

Castillo Añazco, R. y Rosero Moncayo, J. (2015). Empleo y condición de actividad en Ecuador. Ecuador en cifras. Recuperado de http://www.ecuadorencifras.gob.ec/documentos/web-inec/Estudios\%20 e\%2oInvestigaciones/Trabajo_empleo/3.\%20REM-Empleo_condact.pdf

Dubet, F. (2016). ¿Por qué preferimos la desigualdad? Buenos Aires, Argentina: Siglo Veintiuno Editores. Eagleton, T. (2010). Sobre el mal. Titivillus ePub base r1.2.

Instituto Nacional de Estadística y Censos (INEC) (2017). Encuesta de Empleo, Desempleo y Subempleo Urbano y Rural, junio 2017. Recuperado de http://www.ecuadorencifras.gob.ec/ banco-de-informacion/

Instituto Nacional de Estadística y Censos (INEC) (s. d.). Encuesta Nacional de Ingresos y Gastos de los hogares rurales y urbanos 2011-2012. Resumen metodológico y principales resultados. Quito, Ecuador: (s. d.).

Larrea, C. (2006). Hacia una historia ecológica del Ecuador. Propuestas para el debate. Quito, Ecuador: Corporación Editora Nacional.

Larrea, C. y Green, N. (2015). De la lucha contra la pobreza a la superación de la codicia. Ecuador: inequidad social y redistribución del ingreso. En La osadía de lo nuevo. Alternativas de política económica. Quito, Ecuador: Fundación Rosa Luxemburgo.

Laurie, A. (1998). Informe de la Comisión del Subempleo de la 16. ${ }^{a}$ CIET. (s. d.).

Marini, R.-M. (2008). Dialéctica de la dependencia. América Latina, dependencia y globalización. (s. d.). Superintendencia de Control de Mercados. (2013). Indicadores de concentración. Quito, Ecuador: Superintendencia de Control de Mercado. 
I36 Revista Economía | II2 | noviembre 20I8

Villacís, B. (2016). Sobre los nuevos cambios del INEC en las categorías de empleo. Bayron Villacís. Recuperado de https://byronvillacis.org/2016/o7/24/sobre-los-nuevos-cambios-del-inec-en -las-categorias-de-empleo/?blogsub=confirming\#subscribe-blog 